\title{
THE RHIC WALL CURRENT MONITOR SYSTEM*
}

\author{
P.R. Cameron $^{\#}$, R.C. Lee, T.J. Shea, J. van Zeijts, BNL, Upton, NY \\ E. Barsotti, J. Crisp, B. Fellenz, FNAL, Batavia, IL
}

\begin{abstract}
The RHIC Wall Current Monitor System will provide data which closely represents the longitudinal profile of bunches in the RHIC ring. This data will be available throughout the machine cycle, from injection through acceleration, transition, transfer to storage RF, and storage. Information which can be derived from this data includes fill pattern, synchrotron motion, longitudinal bunch profile, beam spectrum, and luminosity. The system is similar to that which has operated successfully at the Fermilab Tevatron and Main Ring[1,2,3]. The detectors [4] are broadband resistive wall current monitors. Their signals are sampled and digitized by a high-speed oscilloscope. A Macintosh computer running LabVIEW controls the scope via GPIB, controls system calibration, processes the data, and communicates to the VME-based RHIC Control System via a PCI/MXI/VME interface.
\end{abstract}

\section{INTRODUCTION}

The Relativistic Heavy Ion Collider (RHIC) at Brookhaven National Laboratory consists of two synchrotrons which intersect at six points around the 3.8 kilometer circumference[5]. Ion beams from protons to fully stripped gold will be accelerated and stored. The baseline design has 60 bunches of about $10^{11}$ charges, with an anticipated upgrade intensity of 120 bunches of 2 $\mathrm{x} 10^{11}$ charges. RMS bunch lengths will vary from about $2.5 \mathrm{~m}$ for gold at injection energy to $7 \mathrm{~cm}$ for protons at full energy.

\section{SYSTEM HARDWARE}

System Hardware is shown in Figure 1.

\subsection{The Detectors}

Detectors are installed near the 2 o'clock Intersection Point in each of the two RHIC rings. As shown in Figure 1 , these detectors are fully integrated into the RHIC Control System. A third stand-alone detector has been installed at the 4 o'clock IP, where the signals from the counter rotating bunches will cancel. This detector will be useful for adjusting the collision point, especially during commissioning. Transfer impedance of the detectors is one $\mathrm{ohm}$. Frequency response of this type of detector is typically flat within $3 \mathrm{~dB}$ from a few $\mathrm{KHz}$ to $6 \mathrm{GHz}$. The

*Work supported in part by the US Department of Energy.

\# Email: cameron@ bnl.gov detectors at the 2 o'clock IP are installed with microwave absorbing material on either side of the detector, to absorb energy propagating down the $7 \mathrm{~cm}$ beampipe above cutoff (2.5 $\mathrm{GHz}$ and $3.3 \mathrm{GHz}$ for the lowest TE and TM modes). Signals travel about $70 \mathrm{~m}$ over low-loss $7 / 8$ inch heliax cable from the detectors to the digitizer in the Instrumentation Control Room.

\subsection{Data Acquisition}

Data is acquired by a LeCroy Model 584AL Digital Storage Oscilloscope controlled via GPIB by a Macintosh G3 computer running LabVIEW. The scope specifications include analog bandwidth of $1 \mathrm{GHz}$, maximum sample rate of $8 \mathrm{GS} / \mathrm{sec}$, maximum retrigger rate in segmented mode of $30 \mathrm{KHz}$, data transfer rates of several hundred $\mathrm{KB} / \mathrm{s}$ through GPIB, and $8 \mathrm{MB}$ of waveform memory. This digitizer is the only one currently available which meets all requirements for our WCM system. In addition to the detector signals, the scope also digitizes the RF bucket clock for each ring, permitting the observation of synchrotron oscillations.

\subsection{Interface to the Control System}

Communication between the Macintosh and the VMEbased Control System[6] is accomplished by a National Instruments PCI/MXI/VME interface. This interface provides $32 \mathrm{MB}$ of shared memory in VME, which is read and written to by both the Macintosh and the VME-based Front End Computer (FEC). Scope triggering is accomplished by a VME-based Beam Synchronous Trigger Module[7]. Timestamps are generated by a VME-based Utility Module and the Trigger Module. Communication with console level computers is accomplished via 100 Mbit/s Ethernet.

\section{SYSTEM SOFTWARE}

System Software resides in the locations shown in Figure 1 .

\subsection{The Application}

Application programs running on Console Level Computers are the Control System's interface between the users (typically operators and accelerator physicists) and programs called Accelerator Device Objects (ADOs) which run in the FECs. The WCM user interface includes fields for setting data acquisition parameters and a graphic display to present the monitored profile data and bunch fill patterns. The application will be integrated with a logging system and will include an option for tomographic 


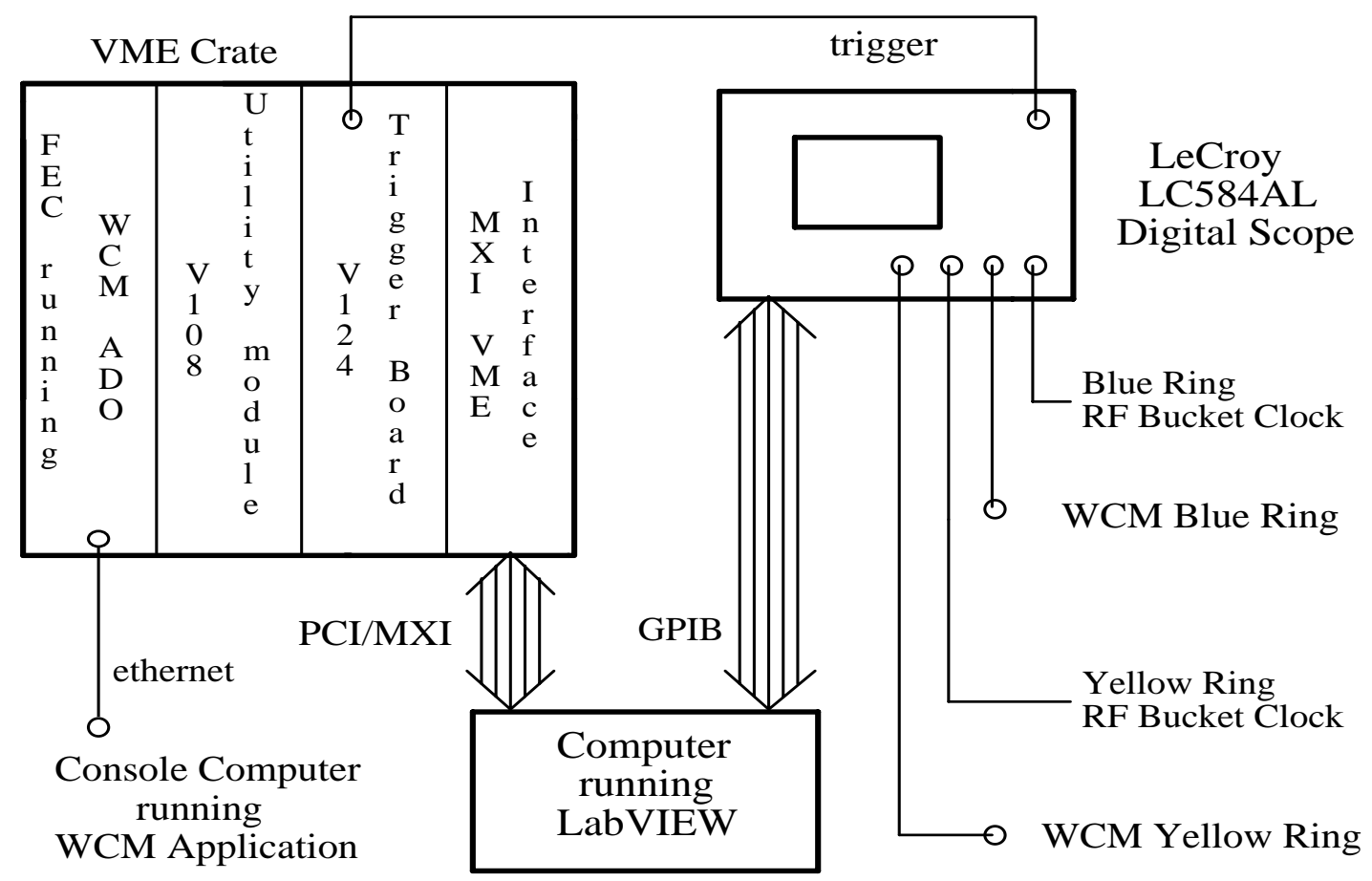

Figure 1: System Block Diagram

reconstruction of the longitudinal phase space using the TOMO package developed at CERN[8].

\subsection{The ADO}

ADOs are the Control System's interface to all accelerator equipment in RHIC. Application programs communicate with ADOs running in FECs. The FECs are VME based Power PC processors using the vxWorks real-time operating system. Data is transferred over $100 \mathrm{Mbit} / \mathrm{s}$ Ethernet using TCP/IP and RPC protocols.

The ADOs for the two WCMs (one for each of the two counter-rotating rings) communicate with the WCM computer through shared VME memory. The memory is divided equally for the two ADOs. The memory for each ADO is partitioned into four blocks - command, status, data control, and data blocks. The data blocks are circular buffers. Commands are sent to the WCM computer which in turn responds with status information. The data control block is used to define the dynamically allocated circular data buffers and to control writing by the WCM computer and reading by the ADO. Semaphores are used in all blocks to synchronize and prevent race conditions when the two computers access memory.

ADO parameters allow application programs to define the type of acquisition - sample rate, profile length, number of profiles, scope scale and offset, and trigger requirements. Beam-synch clock event decoder modules for each ring are set up by the ADO to provide triggers to the WCM scope. Timestamps associated with each acquisition are provided by the ADO to aid data correlation with other accelerator events. Data is transferred to applications from the ADO either on demand or automatically when an acquisition is complete.

\subsection{The LabVIEW Program}

The possibility of controlling the scope in $\mathrm{C}++$ directly from the ADO was considered and rejected based upon the need for calibration and the availability of existing calibration, instrument control, and analysis software.

Calibration of bunch intensities and width is based upon the system frequency response and the beam spectrum. The frequency response of the detector, cabling and connectors, and oscilloscope signal path is measured. The detector can be readily measured only before tunnel installation. The rest of the path can be measured periodically with a calibrated signal generator at relevant frequencies and gains, resulting in a transfer function with two dimensions, frequency and gain. An off-line simulation program then transmits an ideal gaussian representing beam through this transfer function to generate the intensity and width calibration factors.

There are instances where local processing of data either in the scope or in LabVIEW before transfer to the Control System is advantageous. Bunch fill patterns require only that a single number, the intensity of each bunch, be made available in the control room. Powerful scope-based timing and jitter analysis (which might ease analysis of synchrotron oscillations) is available, as are frequency domain transforms. LabVIEW has an extensive suite of 
well-developed data analysis software. The balance between local and console level analysis is evolving.

\subsection{Acquisition Modes}

A variety of acquisition modes are necessary for RHIC acceleration and storage. Some of those which we intend to have available for day one operations are shown in the following table. With the RHIC $78 \mathrm{KHz}$ revolution frequency and the $30 \mathrm{KHz}$ maximum scope retrigger rate, the value for $\mathrm{N}$ in the table below must be 3 or greater.

Table 1: Acquisition Modes

\begin{tabular}{|l|c|c|c|}
\hline Mode & $\begin{array}{c}\text { No. of } \\
\text { Samples }\end{array}$ & $\begin{array}{c}\text { Sample } \\
\text { Length } \\
{[\text { KB ] }}\end{array}$ & $\begin{array}{c}\text { Record } \\
\text { Length } \\
{[\text { KB] }}\end{array}$ \\
\hline $\begin{array}{l}\text { Injection - digitize } \\
\text { continuously for } \\
\text { sample length }\end{array}$ & 1 & $\begin{array}{c}200 \\
\text { typically }\end{array}$ & 200 \\
\hline $\begin{array}{l}\text { Multi-turn - digitize 1 } \\
\text { turn every N turns }\end{array}$ & $\mathrm{N}$ & 20 & $20^{*} \mathrm{~N}$ \\
\hline $\begin{array}{l}\text { Multi-bunch - digitize } \\
\text { 1 bunch every N turns }\end{array}$ & $\mathrm{N}$ & 0.1 & $0.1^{*} \mathrm{~N}$ \\
\hline
\end{tabular}

\section{OTHER POSSIBILITIES}

The existence of a flexible and powerful data acquisition system at the commissioning of a new and unique machine opens new opportunities.

The unprecedented combination of beam current and particle charge present with gold beams in RHIC opens the possibility of broadband observation of the longitudinal Schottky signal. To the best of our knowledge, all previous observations of the Schottky signal have been with resonant detectors. A broadband Schottky monitor would be an excellent diagnostic for the mysterious 'micro-coherent' signal which has been seen at all other high energy accelerators[9], and which has frustrated efforts to implement stochastic cooling. With high pass filtering and perhaps some pre-amplification, the WCM system might provide broadband Schottky spectra.

Similarly, by utilizing a detector sensitive to transverse position, broadband observation of the transverse Schottky signal might be possible, perhaps with the data acquisition being the same. These broadband spectra might provide useful information about chromaticity. Such a system might also be useful for chromaticity measurements by observing the coherent betatron tune shift between the head and tail of the bunch[10].

\section{CONCLUSIONS}

Detectors have been fabricated and installed. A flexible and powerful data acquisition system has been assembled, and integration has been demonstrated from the scope through to the console level application. We await beam with eagerness and enthusiasm.

\section{REFERENCES}

[1] C.D.Moore et.al., "Single Bunch Intensity Monitoring System Using an Improved Wall Current Monitor", Proc. 1989 PAC, P. 1513.

[2] E.L. Barsotti, "A Longitudinal Bunch Monitoring System Using LabVIEW and High-speed Oscilloscopes", Proc. 1994 Beam Instrumentation Workshop, Vancouver, AIP 333, P.466.

[3] an online description of the FNAL Sampled Bunch Display is available at

http://www-rfi.fnal.gov/SBD/systems.html

[4] R.C.Webber, "Longitudinal Emittance: An Introduction to the Concept and Survey of Measurement Techniques Including Design of a Wall Current Monitor", Proc. 1989 Beam Instrumentation Workshop, Brookhaven, AIP 212, P.85.

[5] http://www.rhichome.bnl.gov/RHIC/index.html

[6] T. S. Clifford, D. S. Barton and B.R. Oerter, "The Relativistic Heavy Ion Collider Control System", Proc. Int. Conf. on Accelerator \& Large Experimental Control Systems (ICALEPCS '97), IHEP, Beijing, China, November 3-7, 1997, P. 12.

[7] H. Hartmann and T. Kerner, "RHIC Beam Synchronous Trigger Module", these proceedings.

[8] S. Hancock, P. Kanus, and M. Lindros, Tomographic Measurement of Longitudinal Phase Space Density, submitted to Elsevier Preprint.

[9] D.A. Goldberg, private communication.

[10] H. Schmickler, private communication. 